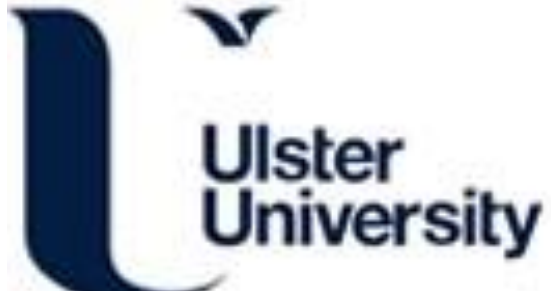

\section{Mixed e-learning and virtual reality pedagogical approach for innovative hydrogen safety training of first responders}

Tretsiakova-McNally, S., Maranne, E., Verbecke, F., \& Molkov, V. (2017). Mixed e-learning and virtual reality pedagogical approach for innovative hydrogen safety training of first responders. International Journal of Hydrogen Energy, 42, 7504-7512. https://doi.org/10.1016/j.ijhydene.2016.03.175

Link to publication record in Ulster University Research Portal

\section{Published in:}

International Journal of Hydrogen Energy

Publication Status:

Published (in print/issue): 16/03/2017

DOI:

10.1016/j.ijhydene.2016.03.175

\section{Document Version}

Author Accepted version

\section{General rights}

Copyright for the publications made accessible via Ulster University's Research Portal is retained by the author(s) and / or other copyright owners and it is a condition of accessing these publications that users recognise and abide by the legal requirements associated with these rights.

\section{Take down policy}

The Research Portal is Ulster University's institutional repository that provides access to Ulster's research outputs. Every effort has been made to ensure that content in the Research Portal does not infringe any person's rights, or applicable UK laws. If you discover content in the Research Portal that you believe breaches copyright or violates any law, please contact pure-support@ulster.ac.uk. 


\title{
MIXED E-LEARNING AND VIRTUAL REALITY PEDAGOGICAL APPROACH FOR INNOVATIVE HYDROGEN SAFETY TRAINING OF FIRST RESPONDERS
}

\author{
Tretsiakova-McNally, S. ${ }^{1}$, Maranne, E. ${ }^{2}$, Verbecke, ${ }^{3}{ }^{3}$ and Molkov, ${ }^{1}{ }^{1}$ \\ ${ }^{1}$ HySAFER Centre, Ulster University, Shore Road, Newtownabbey, BT37 0QB, Northern \\ Ireland, UK, s.tretsiakova-mcnally@ulster.ac.uk and v.molkov@ulster.ac.uk \\ ${ }^{2}$ CRIsis Simulation Engineering (CRISE), Parc Club du golf, bat.7, Aix-en-Provence, 13855, \\ France, eric.maranne@vr-crisis.com \\ ${ }^{3}$ QHSE Department, AREVA Stockage d'Energie, Avenue Louis Philibert - CS 10656 \\ 13547 Aix-en-Provence Cedex 4, France, franck.verbecke@areva.com
}

\begin{abstract}
Within the scope of the HyResponse project (www.hyreponse.eu) the development of a specialised training programme is currently underway. Utilizing an andragogy approach to teaching, distance learning is mixed with classroom instructors-led activities, while hands-on training on a full-scale simulator is coupled with an innovative virtual reality based experience. Although the course is dedicated mainly to first responders, provision has been made to incorporate not only simple table-top and drill exercises but also full-scale training involving all functional emergency response organisations at multi-agency cooperation level. The developed curriculum includes basics of hydrogen safety, first responders' procedures and incident management expectations.
\end{abstract}

Keywords: hydrogen safety, first responders, training.

\subsection{INTRODUCTION}

Fuel Cell and Hydrogen (FCH) 1 technologies and applications both in transport and energy sectors arrive to the market today. Unfortunately at the moment, European fire authorities and first responders have limited knowledge of these new technologies. Thus, an adequate training of first responders should provide them with an accurate, up-to-date knowledge of hydrogen safety basics and with essential practical skills on how to handle potential incidents/accidents within FCH systems and infrastructure; on how to protect the general public without putting lives at risk. The Coordination and Support Action project HyResponse, funded by the European Commission Fuel Cell and Hydrogen Joint Undertaking (FCH JU), aims to establish the first comprehensive training programme - European Hydrogen Safety Training Platform (EHSTP)2 [1]. The project partners are French Academy for Fire, Rescue and Civil Protection Officers (ENSOSP), Air Liquide, Ulster University (UU), FAST/EHA (European Hydrogen Association), CCS Global group, CRIsis Simulation Engineering (CRISE) and AREVA Stockage d'Energie.

The target audience of the EHSTP are first responders including fire-fighters at different levels, from field operators to high-level incident commandment, police forces, paramedics, vehicle recovery personnel, site operators, etc. The EHSTP consists of three key elements: educational training (i.e. lectures), operational exercises and innovative virtual reality (VR) 3 exercises, which reproduce in detail an entire accident scenario including either correct or incorrect intervention techniques. The educational training encompasses the basics of hydrogen safety; regulations, codes and standards (RCS)4; the intervention strategies and tactics relevant to first responders.

This paper describes the development of the HyResponse lectures and VR exercises through the use innovative VR approaches combined with a comprehensive pedagogic toolbox:

\footnotetext{
1 FCH - Fuel Cell and Hydrogen

2 EHSTP - European Hydrogen Safety Training Platform

3 VR - Virtual Reality

4 RCS - Regulations, Codes and Standards
} 
- different types of VR exercising;

- face-to-face teaching and distant learning through lectures, group discussions, problemsolving tasks, seminars and independent studies;

- a physical simulator for hands-on classroom training;

- face-to-face teaching and distant learning, testing, validation and assessment tools;

- face-to-face and distant VR tools for virtual hands-on drilling and assessment, either on individual or collaborative levels.

\subsection{PEDAGOGIC SCOPE AND CONCEPTS}

During the development of the HyResponse educational materials and VR exercises the pedagogic concepts of andragogy, i.e. the adult learning theory, have been taken into consideration [2]. The andragogy focuses on a self-directed learner. It requires less didactic and more hands-on approaches with real-life applications, and is based on previous and acquired experiences.

According to Knowles et al. (1984) there are four principles applicable to an adult-centred learning:

1. Adults need to be involved in the planning and evaluation of their instruction.

2. Experience (including mistakes) provides the basis for learning activities.

3. Adults are most interested in learning subjects that have immediate relevance to their job or personal life.

4. Adult learning is problem-centered rather than content-oriented [2].

Besides, an adult learner brings into the continuing educational arena a rich array of experiences that will affect the learning styles and assimilation of knowledge. All these principles are highly relevant to the education, experience acquisition and dissemination activities of the HyResponse project.

An attainment of the first principle is an organisational matter, and the implementation of the HyResponse training sessions will require an active participation of trainees in the evaluation of all the educational materials produced in order to improve them [1]. It is expected that the HyResponse audience will understand why the programme is important to their learning and life situations. The trainees will be able to apply the knowledge they gain during the training to their own job situations and professional life, thus leading to an accomplishment of the third andragogy principle. The addressing of the above mentioned points no. 2 and 4 are the essence of this paper in particular, and are the cornerstones in the development of high quality hydrogen safety training for first responders in general.

Hydrogen risk is a technological risk; hence it calls for a deeper understanding of the main safety principles and features of FCH systems and infrastructure. Adult learners need to be convinced, and the technological elements are to be explained in detail at the audience cognitive level.

As per Knowles et al. [2], training objectives should be 'SMART':

- Specific: clear and easy to understand

- Measurable: able to be quantified

- Achievable: able to be attained

- Realistic: true to life

- Task oriented

Within the scope of the HyResponse project these objective consider:

- An evaluation of a first responder agency's current state of emergency readiness to deal with hydrogen specific behaviors during incidents/accidents.

- Knowledge gaps, weaknesses, or areas of concern that might affect the agency's performance.

- A level of first responders' knowledge and understanding of hydrogen emergency preparedness roles and responsibilities.

- An applicability of current readiness to emerging hydrogen safety problems. 
With this in mind, the main objectives of the educational segment of the HyResponse programme are as follows:

- to provide first responders with the awareness and understanding of the specificities of hydrogen as an energy carrier during its production, transportation, delivery and uses;

- to familiarise first responders with the operational principles and safety aspects of a range of FCH applications including FC vehicles, refuelling stations, back-up power generation and stationary fuel cell systems for combined production of heat and power;

- to develop a critical evaluation of the safety issues associated with hydrogen physical and combustion properties;

- to achieve a systematic understanding of why the safety of hydrogen differs from that of conventional fuels;

- to provide first responders with a knowledge of potential hazards, relevant safety concepts and safety features for targeted FCH systems and infrastructure;

- to ensure that first responders are aware of typical risk scenarios for FCH systems and infrastructure;

- to instruct first responders on the correct intervention strategy and tactics on how to handle potential consequences of incidents/accidents at $\mathrm{FCH}$ systems and infrastructure;

- to develop in first responders an ability to deal in real life situations with hazardous phenomena involving accidental hydrogen release, hydrogen fires and explosions by applying the principles of hydrogen safety engineering;

- to provide first responders with an analysis of hydrogen safety approaches and requirements defined in RCS related to FCH systems and relevant to the appropriate actions to be taken by first responders;

- to provide first responders with a clear picture of the safety requirements prescribed in RCS with respect to mitigation measures; assessment of the incident/accident scenes; safety strategies for the operation of first responders at the scene of an accident.

Although the lectures are very important to overcome biases and preconceptions, there is a need for adults to have a real hands-on experience. This is achieved through a physical simulation platform available at ENSOSP premises. While the initial training sessions will definitely need this physical set-up, a widespread use of FCH technologies in the coming years will lead to the basic technical knowledge and acquired experience of the public.

The second andragogy principle implies that the training should be more task-oriented as opposed to simple memorization of facts, i.e. the learning/teaching activities should be in the context of the common tasks to be performed by first responders. To address this need, we will demonstrate in the following sections how the VR aligned to the lectures content can be used to achieve the taskorientation, and how the VR will be used to provide experience acquisition through problem-solving approaches. Different types of problem-centred exercises will be described in the paper. Their purpose is to overcome inhibitions; to provide individual and shared experiences; and to take into account the wide range of learners' backgrounds, the diversity of institutions and cultures (nations), and to allow for different levels/types of previous learning and professional experience. There are some challenges the tutors faced during the design and development of educational content of the EHSTP. In a short term (during the face-to-face training sessions) the learners will represent the knowledgeable and motivated group of first responders (so-called 'elite), while in the long run the distant learning transmitted via the online training course will target all levels of emergency personnel and even the general public. Therefore, it is very important to tailor the teaching materials as to meet the diverse demands and expectations of the HyResponse audience.

The VR technology gives an ample opportunity to address several expectations of the andragogy theory:

- an explanation and explicitness: the production of real-life foreseen problems of hydrogen wide use enables to picture and explicitly expose why specific protocols, functions, operations, etc. are needed;

- task-orientation and interaction: the VR is the perfect tool for this; 
- relevance and real/personal life relation will have to be designed through the choice of environments, operational problems, and scenarios;

- the low cost of VR scenarios creation enables to provide a rich set of situations aligned to first responders diverse experiences, as extensively as possible;

- the VR can accommodate the training schemes ranging from a single user to full size multiagency set-ups providing many inhibition avoidance strategies, and gives a fresh approach to learning that may overcome existing beliefs or reluctance.

\subsection{TRAINING ARTICULATION}

The HyResponse training utilises a blended learning approach. The trainees will attend a typical 'brick-and-mortar' school-type structured sessions, where instructors-led lectures are combined with the VR exercises and supported with hands-on operational training activities. The developed educational materials in their final versions will be made available to the public through the HyResponse website, thus allowing the trainees to control the time, place, route, or pace of their postclassroom studies. In addition, the interactive online training course will include videos of demonstrations and experiments, photos, links to other website and other sources of information, etc.

\subsection{The International Curriculum and Lectures}

The educational training aims to provide first responders with the fundamental knowledge on hydrogen safety prior to the operational and virtual reality exercises. The International Curriculum (IC)5 on hydrogen safety training for first responders forms a foundation of the underpinning educational materials for face-to-face training sessions, and provides an outline for the online training course. The approaches to the IC development and the learning needs of first responders have been discussed with the internal and external international experts from HySAFER centre (Ulster University, UK), California Fuel Cell Partnership (USA), Pacific Northwest National Laboratory (USA), Fuel Cell Technologies Office (DoE, USA), the Commission for Extrication and New Technology (Belgium), State Fire Department and Emergency Medical Service Hamburg (Germany), and Research Centre for Fire Protection (Poland).

During the development of the research-led IC a thorough review of more than 400 literature and webbased sources was carried out to reflect the state-of-the-art in hydrogen safety science and engineering. The IC encompasses all topics relevant to first responders' educational needs. There are three sections in the IC: 1) Basics of Hydrogen Safety; 2) Regulations, Codes and Standards; 3) Intervention Strategies and Tactics for first responders. The first section on Basics of Hydrogen Safety contains nine modules and covers the following themes: overview of FCH applications, infrastructure and uses; specific safety issues related to hydrogen production and storage; overview of incidents/accidents involving hydrogen; basic properties and behaviour of hydrogen (including combustion); comparison of hydrogen to other common fuels; interactions and compatibility of hydrogen with different substances; hazards and risks associated with gaseous and liquefied hydrogen; hydrogen releases; ignition of hydrogen-oxidizer mixtures; hydrogen flames and fires; hydrogen releases indoors; explosions and blast waves; prevention, mitigation and fire-fighting techniques. The second section of the IC is devoted to RCS and requirements to FCH systems relevant to first responders. The third section consists of one module, which introduces typical accident scenarios for selected FCH applications; relevant emergency response strategies and tactics; and rescue operations. The IC also includes the essential (10 references) and the further (378 references) reading lists that both first responders and tutors will use for learning and teaching purposes. Each topic within a module is provided with the appropriate reference(s) to facilitate the ease of finding necessary resources during the independent learning. In addition, the IC contains a glossary, a schedule for the face-to-face training sessions and a list of relevant RCS. The draft of the IC can be found on: http://www.hyresponse.eu/curriculum.php [3].

\footnotetext{
5 IC - International Curriculum
} 
The primary goals of the HyResponse lectures are to stress the importance of hydrogen as a new energy carrier, to focus on the specificities/hazards of hydrogen compared to traditional fuels (natural gas, petrol, propane, etc.) and to introduce first responders to new $\mathrm{FCH}$ systems, infrastructures, associated risks, accident scenarios, and RCS. The lectures are aligned to the IC and extensively supported by the examples of incidents and/or accidents reported in the media, on the YouTube channel, in European HIAD [4] and on the US DoE Hydrogen Lessons Learned [5] databases. The web-based resources available from the relevant projects such as HySafe [6], HYPER, H2FC [7], HySAFEST, HyCourse, TrainHy-Prof [8], HyFacts [9], HyIndoor [10] and the related courses: MSc Hydrogen Safety Engineering at Ulster; US DoE Introduction to Hydrogen Safety for First Responders [11] supported the development of the educational materials.

A brief description of the planned HyResponse lectures is given below:

- Introduction to FCH applications and hydrogen safety principles

- Hydrogen properties relevant to safety

- Safety of hydrogen storage

- Harm criteria for people and damage criteria for structures

- Unignited hydrogen releases, their prevention and mitigation

- Hydrogen ignition sources and prevention of ignition

- Hydrogen fires

- Dealing with hydrogen explosions

- Hazards of hydrogen use indoors and relevant mitigation techniques

- RCS for first responders

- Intervention strategies and tactics for first responders

The lectures will include but not be limited to the following topics: an overview of hydrogen production, storage, transportation, delivery and uses in the modern world; descriptions of FCH systems and infrastructure; safety features of FCH applications; basic hydrogen properties; hydrogen storage options; compatibility of hydrogen with different materials; hazards and events that may lead to fires, loss of life and cause damage to structures, equipment and environment; typical incidents/accidents scenarios; ignited and unignited hydrogen releases; possible ignition sources and prevention of ignition; combustion of flammable hydrogen-air mixtures; separation from hydrogen flames and firefighting; hydrogen deflagrations and detonations; European guidelines for inherently safer use of FCH systems indoors; identification and mitigation measures as well as RCS, intervention strategies and tactics.

All the materials are designed with the view to enhance a deep understanding of hydrogen-related phenomena by the first responders having different professional backgrounds. The lectures are prepared using Microsoft Power Point and Word applications. They contain a variety of visual information (such as schemes, figures, diagrams, graphs and movies) and the embedded links to the sources where additional information can be easily found. An example of lecture slides is shown in Figure 1.

The educational materials are very interactive. Almost every lecture contains a number of videos or animations that will help to visualise some processes, scenarios, catastrophic events, etc., and to explain some technically difficult concepts. To achieve the forth andragogy principle of problemcentred learning it is planned that during classes the trainees will execute tasks using Cyber Laboratory engineering tools [12]. These tasks are aligned to the operational and VR training exercises for the specific incidents/accidents scenarios. For example, the first responders will be asked: to assess the size of hydrogen flammable envelope; to calculate hydrogen jet parameters; to evaluate a hydrogen tank blowdown time; to calculate flame lengths and deterministic separation distances; to assess the consequences of pressure peaking phenomenon, etc. 
Atomic and molecular hydrogen

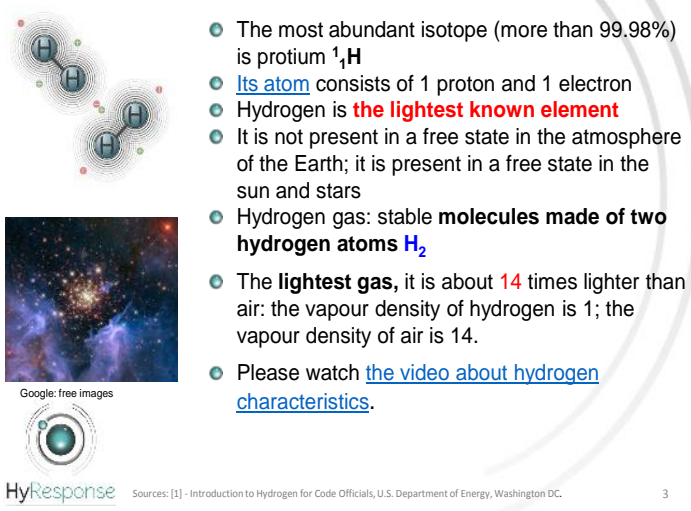

What is a Fuel Cell (FC)?

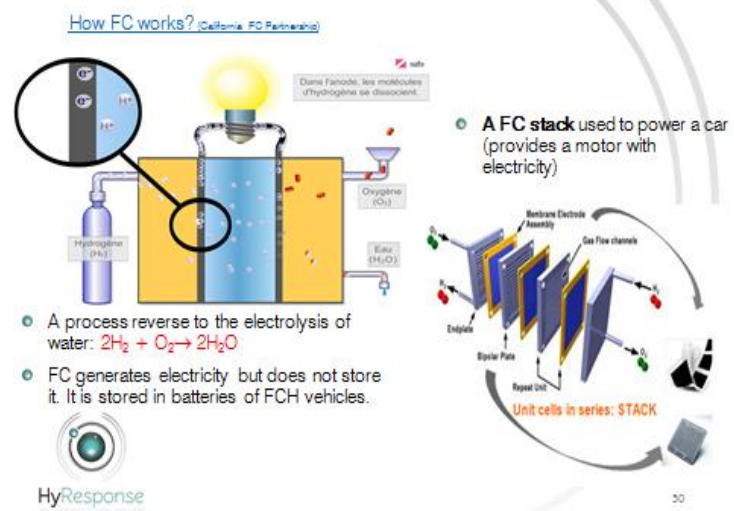

Figure 1. An example of the slides for HyResponse lectures.

The lecture slides also provide a pedagogic support to the lecturers during the face-to-face sessions. The preparation of the lecture notes (to be presented as text documents) is currently underway. All the educational materials will be integrated into the online training course in the end of the project, and will eventually form the HyResponse educational wallet.

\subsection{Virtual Reality Exercises}

An exercise is a generic term for a range of activities that tests emergency response readiness, evaluates an emergency response plan, and can assess the success of training and development programmes. The typical purposes of the VR exercises are:

- to validate emergency plans as well as the curriculum effectiveness during and after pilot training sessions - validation;

- to develop first responders competencies and to give them practice in carrying out their roles, new doctrinal approaches, standard operating procedures $(\mathrm{SOPs})_{6}$, and rules of engagement (ROEs) 7 - training;

- to develop an understanding of organizational roles as well as hydrogen specific risks, interrelationships and inter-dependencies - learning;

- to test the established procedures - testing.

There are five basic categories of VR exercises:

- orientation exercises,

- tabletop exercises (TTXs)8,

- drills,

- functional exercises (FEs)9,

- full-scale exercises (FSEs) 10.

The first two categories of exercises are primarily discussion-based, whereas the other three are action-oriented. It should be noted that these categories and purposes of the exercises are overlapping and inter-related, depending on the type of the audience to be trained. Thus, it is also necessary to define who will participate in the exercises. As for the HyResponse project, first responders can be

\footnotetext{
6 SOP - Standard Operating Procedure

7 ROE - Rules of Engagement

8 TTX - Tabletop Exercise

${ }_{9} \mathrm{FE}$ - Functional Exercise

${ }_{10}$ FSE - Full Scale Exercise
} 
pictured as those who first arrive to a scene of an accident with a decision task: fire-fighters, police forces, paramedics, industrial experts, etc. Another feature of the targeted audience is that in the coming years we are likely to train seasoned professionals, already in the field, with a strong operational background and risk mitigation experience. The number of participants, both as trainees or trainers, would also impact on the aim, objectives of the VR exercises.

\subsubsection{Orientation Exercises}

These types of exercises are to be carried out during the lectures, workshops, and seminars. The purposes of an orientation exercise are to:

- familiarize novices with risks, emergency ROEs, standard operating procedure SOPs;

- familiarize experienced first responders with new or changing information/procedures.

An orientation exercise can be organized under a variety of circumstances, including the initiation of a new plan, procedure, or mutual aid agreement, or in the event of a new staffing, leadership, facilities, or risk(s). The focus is usually on a single function with roles and responsibilities of participants clearly identified.

For the orientation exercises, a constructive and explicit problem exposure is usually coordinated by one instructor in a face-to-face mode as a task suitable either for an individual or for a homogeneous group of participants. The orientation exercises are designed to guide a learner towards a problem. This should involve a clear explanation why it is necessary to train hydrogen safety discipline, and should include a directing towards the different contents of the proposed training. Ultimately, we wish the public to be self-directing to the HyResponse lectures, so we need to provide the proper orientation. Both the physical and virtual simulators will be used to expose, illustrate, anchor and enforce the lectures contents. It should be noted that the virtual simulation tool will make it easier for the trainees to catch up with new technologies, products, information and procedure changes, as well as to discuss potential impacts of FCH technologies or RCS evolution.

The examples of orientation exercises will include:

- dissemination material on the website (including pictures and VR presentations);

- workshops or seminars presentations;

- an animation of constructive and explicit problem exposure and solving (usually performed by a single instructor in a face-to-face mode to a homogeneous participants group (films, VR presentations, etc.).

\subsubsection{Tabletop Exercises}

A tabletop exercise (TTX) is a low-stress event aimed to trigger a discussion of a simulated situation. The TTX participants are encouraged to discuss issues in detail and to make decisions using slowpaced problem-solving methods driven by the instructor as opposed to the spontaneous, fast-paced ones typical for the actual or simulated emergency conditions. The TTXs are introduced at early stages, in parallel to functional and full-scale exercises. A constructive problem-solving is the goal of such exercises. A copy of the appropriate emergency plan and other pertinent materials are available for the reference during the TTX. A person is assigned to act as a recorder documenting actions taken during the TTX; these notations serve as a reference tool for evaluating the exercise. The TTXs are designed to involve the participants in understanding the problems and the protocols/functions/operations he/she has to deal with. It enables to explicitly understand how these protocols are fitting in their everyday life and organisation. A TTX may involve multi-agencies participants as it enables to understand each other's limitations, expectations, needs and capabilities, forces and weaknesses.

A TTX begins with a briefing by the facilitator as to guide both the participants and simulators to the TTX objectives, ground rules, and communication and simulation procedures. The scenario narrative is then presented in an intelligence briefing. The scenario is generally invented and describes an event 
or emergency incident bringing participants up to a simulated 'present moment' in time. From there, a virtual simulator is used to provide realistic materials and visions suitable to describe the subsequent interactive pacing of events decisions and effects. A TTX should not be perceived as 'real time': some events may be accelerated if not conveying an interest for the discussion, others may be slowed down in order to expose inner mechanisms, or to simply provide enough time for discussion. The constructive and interactive problem-solving is usually performed by one instructor, who teaches a heterogeneous group of participants.

In the frame of the first HyResponse workshop that took place at ENSOSP on the 3-4 September 2014, a simple presentation set-up has been provided as a TTX where the workshop participants played roles of hydrogen 'experts'. The set-up was designed around three virtual access points:

- The first responders team, which had access to the VR from their own point of view, performing proactive information gathering, making sense of the events and mitigating risks under tactical control.

- A tactical control was performed collaboratively and explicitly by the assembly of workshop attendees (TTX set-up) in a different room. They were provided with a projected view issued from a virtual camera (CCTV) located nearby the incident.

- Finally, a virtual helicopter was available for the attendees, ready to fly, with a real pilot at disposal.

Two scenarios were considered during the workshop: a multiple car crash on a motorway involving a FC vehicle (Symbian equipped Kangoo) and an overturned trailer currying liquefied hydrogen positioned near the shopping mall. The conditions and environments of both scenarios are shown in Figures 2 and 3.

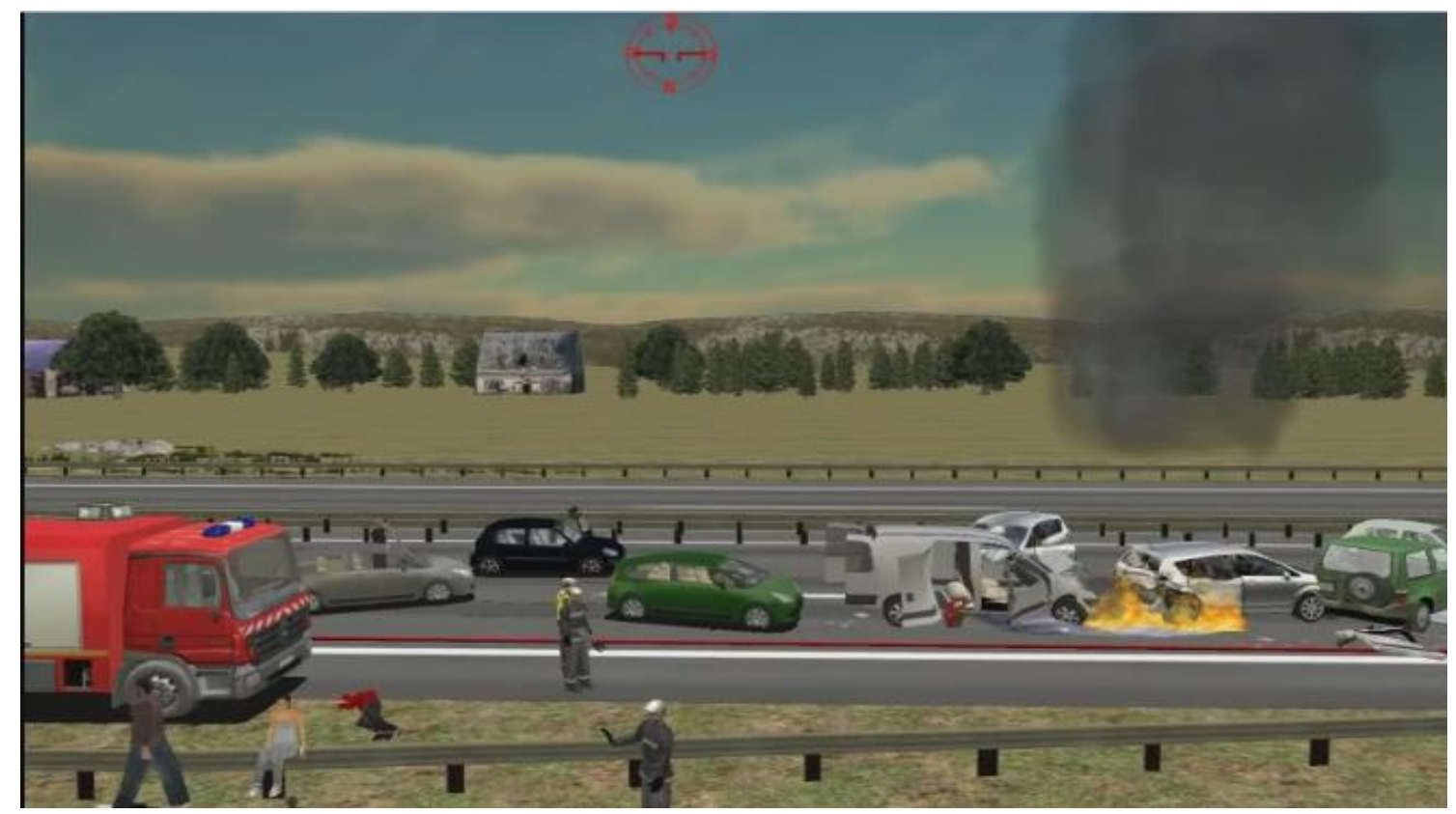

Figure 2. A screenshot of the VR exercise involving a multiple cars crash on a motorway. 


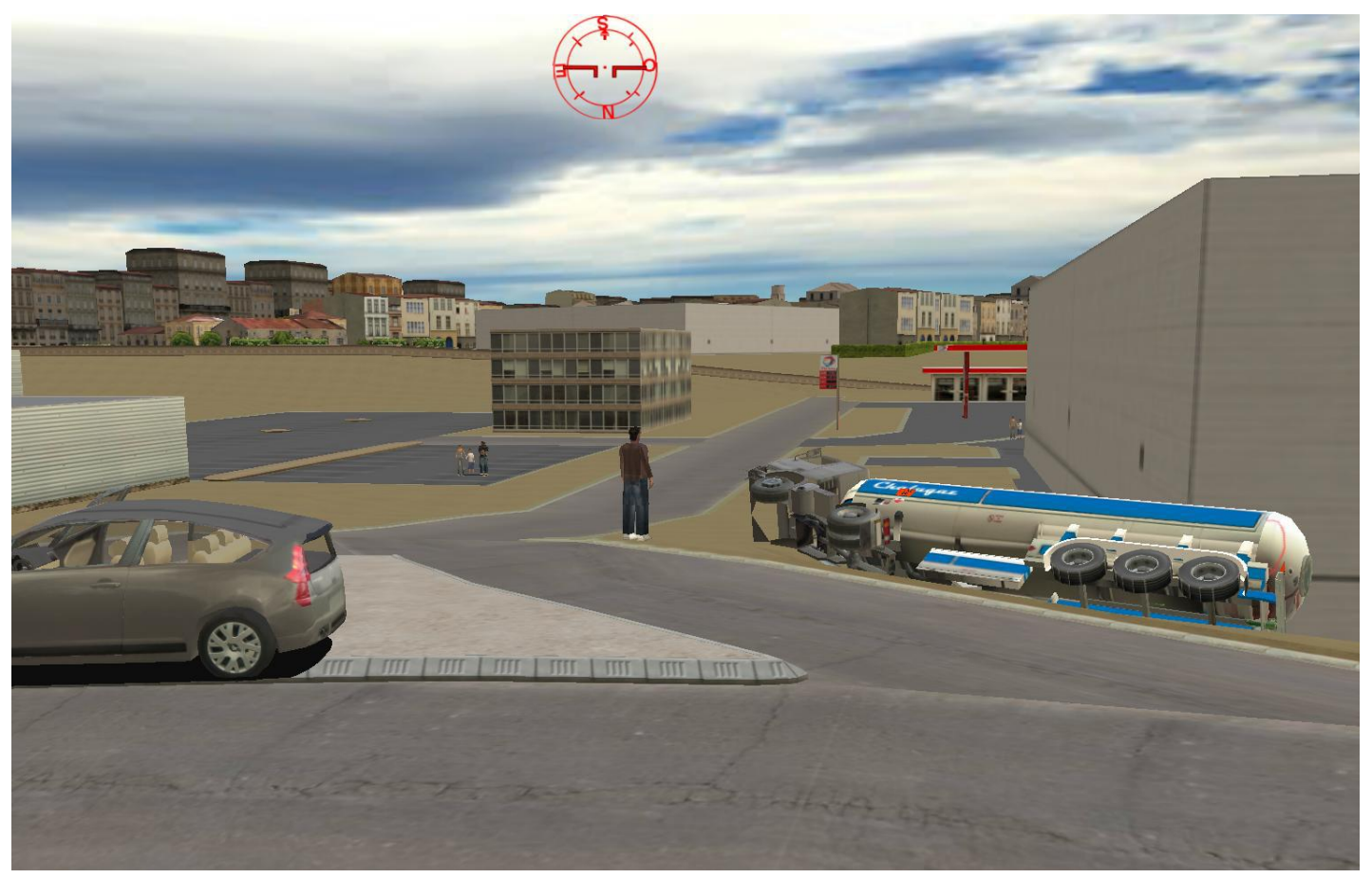

Figure 3. A screenshot of the VR exercise involving an overturned trailer with liquefied hydrogen.

More detailed report of the VR exercises can be found on the workshop web-page: http://www.hyresponse.eu/workshop.php [13].

\subsubsection{Drills}

The main purpose of a drill is to use a repetition to instruct first responders thoroughly. Drills can be used to test and optimise personnel training, response time, inter-agency cooperation and resources, workforce and equipment capabilities. Another purpose of the drills is to put trainees in very different operational environments implying a danger area assessment in a wide variety of situations. For each FCH application (automotive, storage, distribution, production, etc.), the drills allow to test and improve a threat evaluation through a number of slightly different exercises (for example, automotive FCH systems: on the road, in open/underground car parks, in private garages, in a tunnel, in a city centre, etc). This enables to avoid 'task fixation blindness' by practicing situational awareness of an incident scene including personnel, teams, environments, resources and broad picture. Drills ought to induce the importance of situational awareness, personal safety, and personal accountability at the incident scene.

The drills optimally take place after the orientation exercises. First responders should have an understanding of an agency functions that will be tested during the drills and should be given an opportunity to ask questions. Operational procedures and safety precautions are reviewed before the drill begins. Drill categories include but are not limited to reaction, notification, communication, command post, and evacuation. In most cases, a general briefing by the drill designer sets the scene and reviews the drill's purpose and objectives. A drill is usually focused on a segmented functional part of a protocol, like 'immediate response', or 'operative answer'. The VR is used to provide shared operational exposure and understanding; to expose interactive pacing of decisions, actions and effects, and to sustain the drill action with spontaneous or planned events. Physical platform will be used to perform a few drills when possible, but the VR will offer a much broader variety of incidents scenes and rehearsal capabilities. 


\subsubsection{Functional Exercises}

The purpose of a functional exercise is to test and evaluate the capabilities of an emergency response system, or parts of an emergency response system, inter-agency cooperation and resources. The events and situations that would actually occur over an extended period of time are depicted or described. These events and situations are exposed explicitly using the VR or described orally or in writing. Unlike the drills, an FE encompasses more complete system, mixing functional and commandment levels of first responders.

In the context of the HyResponse, the inter-agency level will have to be tested and evaluated as well as coordination and cooperation at the first responders: police forces, medics, fire fighters, industry experts, etc. The VR is used in much the same way as in drills but the scenarios are different and longer, enabling to encompass a function and, commandment-wide, systemic response. An example of FE set-up is given in Figure 4. In this set-up, many trainees are concurrently managing an emergency response. Those trainees may include fire fighters, paramedics, or other kind of first responders (e.g. multi-agency), or a full hierarchical deployment of first responders up to an incident commander including media and other institutions. ENSOSP provides a virtual training facility that may encompass very complex set-ups such as multi-agency, joined forces operations involving multiple commandment levels, from field operators up to high level commandment.

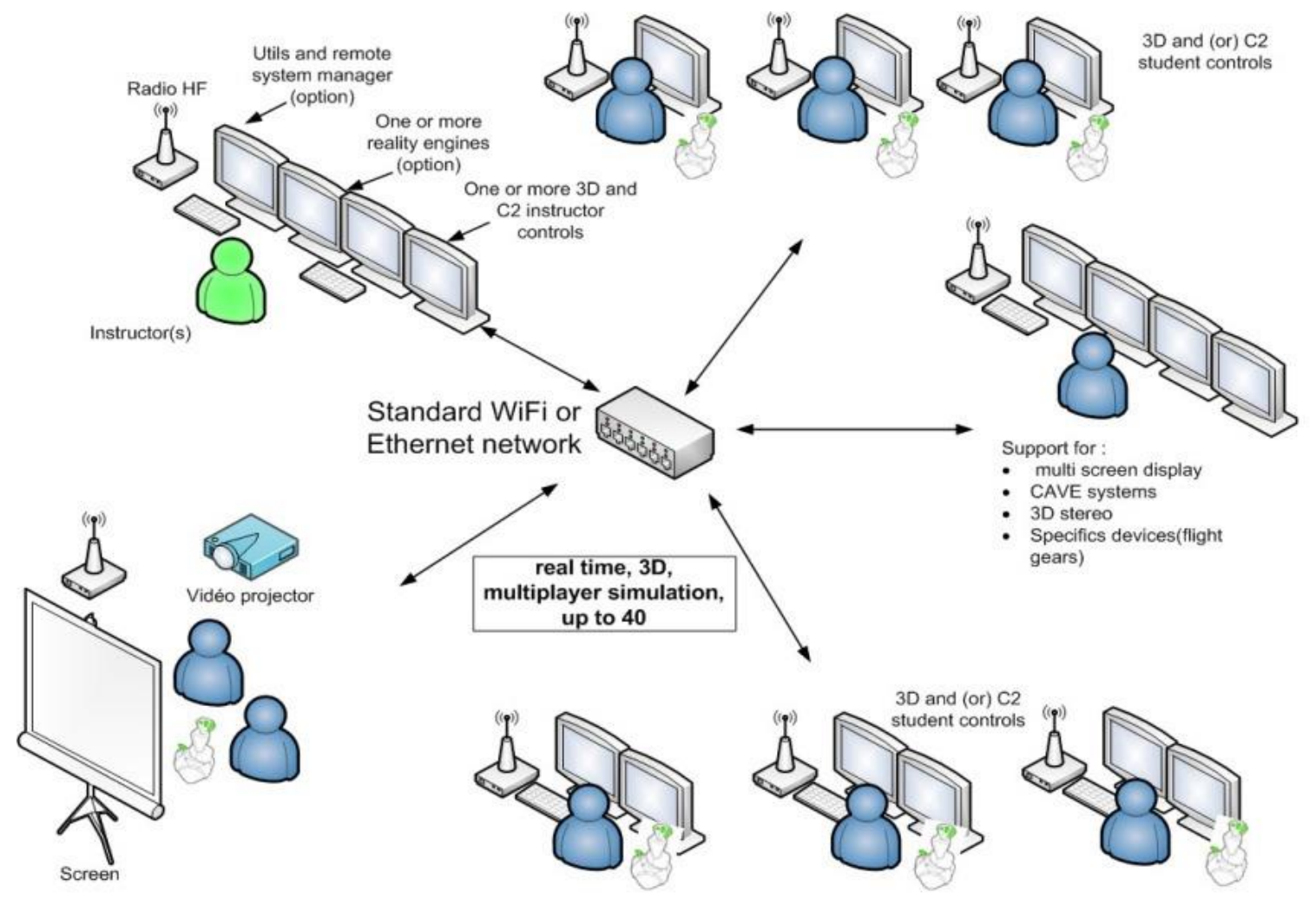

Figure 4. A set-up at ENSOSP for functional training exercises.

\subsubsection{Full-Scale Exercises}

The purpose of FSEs is to test and evaluate a major portion of the emergency operation plan in an interactive manner over an extended time period. The FSEs typically involve more than one agency. Similar to an FE, the objectives of an FSE must be specified, and the actual exercise begins with a simulated event that prompts the initiation of the plan. The FSE includes all of the activities taking place at the emergency operations centre 11 (EOC) as well as on-scene use of simulated victims,

11 EOC - Emergency Operation Centre 
equipment, and workforce. Activities at the scene serve as an input and require coordination with the EOC. An FSE combines the planned and spontaneous messages characteristic for FEs with actions from the field. The goal here is to validate inter-agency cooperation and coordination. The use of the VR is much the same as in the FE exercises. The difference is that other agencies participants' experience, cognitive representation of the events, organisation and response are to be taken into account to build a shared representation of the operational context. The diversity of incident scenarios proposed by the VR framework will enable to run multiple FSEs at low costs.

\section{DISTANCE LEARNING CAPABILITIES}

The developed VR assets can be re-used to compose any scene, and to create any scenario. The VR tool is also used to provide technical, operational and illustrative materials, e.g. in the lectures to depict different types of storages, a fuel cell bus, a fuel cell vehicle, a refuelling station, a storage area with a fuel cell forklift, a small house with a fuel cell vehicle in a garage, etc. The designed VR environments and assets will be used not only for the exercises. They will be integrated as a demonstration for the online course which is composed of:

- a short introduction illustrated with an image gallery from the environment: a leak from a storage, a representation of a separation distance envelope, a representation of the sound, a representation of burning power thresholds, etc;

- a quiz on reflective actions in front of small scenes presented as screenshots;

- a dynamic 3D representation of a leak from a trailer and fire-fighters in action, exported from the VR training tool as 'pure HTML' not necessitating any software download or install on the client machine;

- a short film exported from the VR training tool exposing for example an attack lead on the trailer leak.

The online training course is the work-in-progress and its piloted version has been compiled as a demonstration, as a stand-alone e-pub e-book, readable on any computer or e-book reader (such as Kindle).

\section{CONCLUSIONS}

Current paper highlights the main steps in the development of the European Hydrogen Safety Training Platform within the scope of the HyResponse project. This training programme includes a combination of lectures, virtual and operational exercises. It utilises the mixed e-learning approach with the innovative VR training environments and assets. The international curriculum for hydrogen safety training of first responders has been developed and serves as a basis for the production of the educational materials. The principles on andragogy were applied during the design and development of the Virtual Reality exercises and for the online training course.

\section{ACKNOWLEDGEMENTS}

The research leading to these results has received funding from the European Union's Seventh Framework Programme (FP7/2007-2013) for the Fuel Cells and Hydrogen Joint Technology Initiative under grant agreement No.325348, the HyResponse project "European hydrogen emergency response programme for first responders".

\section{REFERENCES}

1. Verbecke, F., Vesy, B., Lopez, M., Molkov, V., Reijalt, M., Dey, R., Maranne, E., Dang-Nhu, G. European hydrogen safety training platform for first responders: HyResponse project, Proceedings of the Fifth International Conference on Hydrogen Safety, 9-11 September 2013, Paper 233.

2. Knowles, M.S., et al., Andragogy in Action. Applying modern principles of adult education, 1984, Jossey Bass, San Francisco. 
3. HyResponse. International Curriculum on Hydrogen Safety Training for First Responders. Available from: http://www.hyresponse.eu/curriculum.php [accessed on 27.01.16].

4. ODIN: Online Data and Information Network for Energy. Available from: https://odin.jrc.ec.europa.eu/ [accessed on 27.01.16].

5. Hydrogen Tool. Lessons Learned. Available from: https://h2tools.org/lessons/ [accessed on 27.01.16].

6. HySafe - Safety of Hydrogen as an Energy Carrier. Available from: http://www.hysafe.org/ [accessed on 27.01.16].

7. H2FC project. Available from: http://www.h2fc.eu/portal [accessed 08.01.16].

8. Train HyProf project. Available from: http://www.hysafe.org/TrainHyProf [accessed on 27.01.16].

9. HyFacts project. Available from: http://hyfacts.eu/ [accessed on 27.01.16].

10. HyIndoor project. Available from: http://www.hyindoor.eu/ [accessed on 27.01.16].

11. US DoE Hydrogen Safety for First Responders. Available from: https://www.hydrogen.energy.gov/firstresponders.html [accessed on 27.01.16].

12. H2FC CyberLaboratory. http://h2fc.eu/cyber-laboratory [accessed on 08.01.16].

13. HyResponse. First Workshop on Hydrogen Safety Training for First Responders. Available from: http://www.hyresponse.eu/workshop.php [accessed on 27.01.16]. 